\title{
Anticontaminantes alternativos como substitutos ao formaldeído na dieta artificial para criação de Helicoverpa armigera (Hübner, 1805) (Lepidoptera: Noctuidae)
}

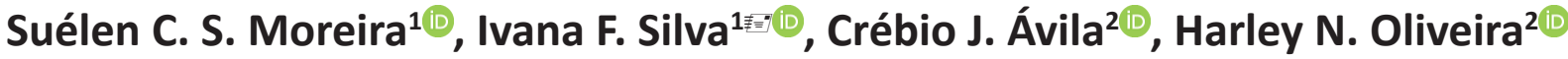

${ }^{1}$ Programa de Pós-Graduação em Entomologia e Conservação da Biodiversidade. Faculdade de Ciências Biológicas e Ambientais, Universidade Federal da Grande Dourados, Dourados, Mato Grosso do Sul, Brasil. ${ }^{2}$ Embrapa Agropecuária Oeste, Dourados, Mato Grosso do Sul, Brasil.

奉" Corresponding author: ivanaf.silva@hotmail.com

Edited by: Daniell R. R. Fernandes

Received: October 18, 2019. Accepted: March 23, 2020. Published: August 07, 2020.

Alternative anti-contaminants as substitute to the formaldehyde in artificial diet for the rearing of Helicoverpa armigera (Hübner, 1805) (Lepidoptera: Noctuidae)

Abstract. Helicoverpa armigera (Hübner, 1805) (Lepidoptera: Noctuidae) is a polyphagous species that has caused damage in several crops in Brazil and worldwide. This work aimed to evaluate anti-contaminants and their respective concentration as follows: Acetylsalicylic acid (1g and $2 \mathrm{~g} /$ diet), sorbic acid (1.85g and 3.7g / diet) and benzoic acid (1.85g and 3.7g / diet) as substitute to the formaldehyde in artificiais diets for the rearing of $\mathrm{H}$. armigera, also having the treatments with formaldehyde and control (no contaminants). Thereby, the effect of the anti-contaminants in the diet over biological parameters of Helicoverpa armigera was evaluated. The highest concentrations of the tested products can replace formaldehyde in diets, since they ensure efficiency in the control of microorganisms. However, among the different anticontaminates studied, benzoic acid in the highest tested concentration $(3.7 \mathrm{~g})$ was considered the most suitable for the rearing of $H$. armigera.

Keywords: Life table, biological characteristics, insect development.

Helicoverpa armigera (Hübner, 1805) (Lepidoptera: Noctuidae) pertence à família Noctuidae e subfamília Heliothinae. Foi constatada, oficialmente, no Brasil no ano de 2013 causando danos em diversos cultivos de importância econômica na região do cerrado brasileiro (Czepak et al. 2013). Os danos causados por essa praga reduzem a produtividade de várias culturas de importância econômica, devido a sua alimentação, especialmente, nas estruturas reprodutivas das plantas, podendo estar dispersa em praticamente todas as regiões agrícolas do país (Ávila et al. 2013). Para a tomada de decisão no controle de $\mathrm{H}$. armigera, informações sobre a bioecologia do inseto podem servir de auxílio no seu manejo para mantê-la abaixo do nível de dano econômico (Razmjou \& Naseri, 2014). A manutenção de criação de inseto em laboratório é imprescindível para o desenvolvimento de estudos entomológicos, básicos e aplicados, essenciais para a elaboração de estratégias efetivas de controle da praga, permitindo maior rapidez nas pesquisas, devido ao suprimento contínuo de insetos para a realização dos experimentos (Parra 2015). Para que isso seja alcançado é necessário a criação de insetos através do fornecimento de dietas artificiais, sendo essa prática essencial para a maioria das espécies de insetos.

Uma dieta nutricionalmente completa deve conter todos, ou pelo menos a maioria, dos elementos necessários ao desenvolvimento dos insetos e deve ser adequadamente balanceada (Smith 1996). As dietas semilíquidas, utilizadas para criação de insetos fitófagos mastigadores, são facilmente contaminadas por micro-organismos e dessa forma, exigem medidas preventivas através do uso de anticontaminantes (Parra 2015). Dentre os agentes químicos mais utilizados como anticontaminantes em dietas artificiais para a criação de insetos destaca-se o uso de formaldeído $\left(\mathrm{CH}_{2} \mathrm{O}\right)$, amplamente utilizado no preparo de dietas artificiais para as criações de diversas espécies, tais como Diatraea saccharalis (Fabricius, 1794) (Hensley \& Hammond 1968), Chloridea virescens (Fabricius, 1977) (Moreti \& Parra
1983), Spodoptera frugiperda (J. E. Smith, 1797) (Kasten et al. 1978), H. armigera (Silva et al. 2018), Anticarsia gemmatalis Hübner, 1818 (Greene et al. 1976) e Diabrotica speciosa (Germar, 1824) (Ávila et al. 2000).

Todavia, essa substância apresenta alta toxicidade para o ser humano, quando manipulada inadequadamente, podendo causar irritação das vias respiratórias, sensibilidade imunológica imediata, mutagênese e até mesmo carcinogênese (Nicron 2017), sendo o risco do uso do formol maior, quanto utilizado em maiores concentrações (ANVISA 2017). Assim, este trabalho tem como objetivo avaliar a eficácia dos anticontaminantes ácido acetilsalicílico, ácido sórbico e ácido benzóico em substituição ao uso do formaldeído na dieta artificial para a criação de $H$. armigera verificando os efeitos em alguns parâmetros biológicos.

Adultos de H. armigera (20 machos e 30 fêmeas), oriundos da criação estoque do laboratório de Entomologia da Embrapa Agropecuária Oeste, foram mantidos em gaiola de tubo PVC $(10 \mathrm{~cm}$ de diâmetro por $22 \mathrm{~cm}$ de altura) fechadas na extremidade inferior com placa gerbox revestida com papel filtro e na extremidade superior fechada com tecido tipo "tule" servindo como substrato para oviposição de $H$. armigera, em condições controladas de temperatura a $25 \pm 1$ oC, umidade relativa de $70 \pm 10 \%$ e fotofase de 12 horas. Posturas foram retiradas e, posteriormente, as lagartas recém eclodidas (24h), oriundas dessas posturas, foram acondicionadas (grupos de 10 neonatas) em tubos de ensaios contendo os diferentes tratamentos $(n=8)$, dietas artificiais baseadas de Greene et al. (1976) (Tab. 1).

Os recipientes (tubos de ensaios) contendo as lagartas foram transferidos para câmaras climatizadas com condições controladas (25 \pm 2 으, umidade relativa de $70 \pm 10 \%$ e fotofase de 12 horas). O desenvolvimento das lagartas foi acompanhado diariamente, registrando-se, a duração e viabilidade da fase larval, além da ocorrência de contaminação das dietas no interior dos tubos de ensaio. 
Tabela 1 . Componentes ( $\mathrm{g}$ ou $\mathrm{mL} /$ dieta) das diferentes dietas utilizadas na avaliação do desenvolvimento de $H$. armigera contendo diferentes anticontaminantes

\begin{tabular}{|c|c|c|c|c|c|c|c|c|}
\hline \multirow{2}{*}{ Componentes } & \multicolumn{8}{|c|}{ Dietas } \\
\hline & 1 & 2 & 3 & 4 & 5 & 6 & 7 & 8 \\
\hline Feijão branco (g) & 112,5 & 112,5 & 112,5 & 112,5 & 112,5 & 112,5 & 112,5 & 112,5 \\
\hline Germe de trigo (g) & 90,0 & 90,0 & 90,0 & 90,0 & 90,0 & 90,0 & 90,0 & 90,0 \\
\hline Proteína de soja (g) & 45,0 & 45,0 & 45,0 & 45,0 & 45,0 & 45,0 & 45,0 & 45,0 \\
\hline Caseína & 45,0 & 45,0 & 45,0 & 45,0 & 45,0 & 45,0 & 45,0 & 45,0 \\
\hline Levedura & 56,3 & 56,3 & 56,3 & 56,3 & 56,3 & 56,3 & 56,3 & 56,3 \\
\hline Ácido ascórbico (g) & 5,4 & 5,4 & 5,4 & 5,4 & 5,4 & 5,4 & 5,4 & 5,4 \\
\hline Solução vitamínica (mL) & 13,5 & 13,5 & 13,5 & 13,5 & 13,5 & 13,5 & 13,5 & 13,5 \\
\hline Metil-p-hidroxibenzoato (g) & 4,5 & 4,5 & 4,5 & 4,5 & 4,5 & 4,5 & 4,5 & 4,5 \\
\hline Tetraciclina 500mg (g) & 0,30 & 0,30 & 0,30 & 0,30 & 0,30 & 0,30 & 0,30 & 0,30 \\
\hline Ágar (g) & 34,5 & 34,5 & 34,5 & 34,5 & 34,5 & 34,5 & 34,5 & 34,5 \\
\hline Água destilada (mL) & 1800 & 1800 & 1800 & 1800 & 1800 & 1800 & 1800 & 1800 \\
\hline Formaldeído (37\%) (mL) & - & 4,5 & - & - & - & - & - & - \\
\hline Ácido acetilsalicílico 500mg (g) & - & - & 1,00 & 2,00 & - & - & - & - \\
\hline Ácido sórbico (g) & - & - & - & & 1,85 & 3,70 & - & - \\
\hline Ácido benzóico (g) & - & - & - & - & - & - & 1,85 & 3,70 \\
\hline
\end{tabular}

${ }^{1}$ Carbonato de cálcio: 21,000; Fosfato de cálcio: 14,900; Sulfato de cobre X 5H2O: 0,039; Fosfato férrico: 1,470; Sulfato de magnésio: 9,000; Sulfato manganoso: 0,020; Sulfato de potássio e alumínio: 0,009; Cloreto de potássio: 12,000; lodeto de potássio: 0,005; Monofosfato de potássio: 31,000; Cloreto de sódio: 10,500; Fluoreto de sódio: 0,057. Niacinamida: 0,5g: Pantotenato de cálcio: 0,5g: Riboflavina: 0,25g: Tiamina: 0,125g; Piridoxina: 0,125g; Ácido fólico: 0,5g; Biotina: 0,02 mg; Vitamina B12 - Clanocobalina 1mg, Água destilada QSP 500ml. Quantidade para 500mL de solução 1-Testemunha; 2-Formaldeido; 3-AAS 1,85g; 4- AAS 3,7g; 5-Ácido sórbico 1,85g; 6-Ácido sórbico 3,7g, 7-Ácido benzóico 1,85; 8-Ácido benzóico 3,7g

A contaminação foi avaliada visualmente, a partir da contagem do número de tubos contaminados. As pupas obtidas foram pesadas 24 horas após sua formação, com auxílio de balança analítica de precisão, e separadas por sexo. Permanecendo individualizadas em copos transparentes descartáveis de polietileno $(50 \mathrm{~mL})$, para avaliação da duração e viabilidade da fase de pupa. Após a emergência dos adultos de $H$. armigera, 20 casais foram individualizados em gaiolas de tubo PVC igualmente descrita acima. A alimentação das mariposas foì feita com mel ( $10 \%$ em solução com água) fornecido através de rolo dental de algodão imerso na solução, o alimento foi diariamente substituído. Foram avaliados diariamente o número de ovos, através da substituição do tule das gaiolas e as durações do período de oviposição e longevidade.

Após a segunda postura de $H$. armigera obtida, 50 ovos de cada casal, foram separados e acondicionados em recipientes plásticos tipo gerbox, para avaliação da duração diariamente e viabilidade da fase de ovo. Durante a fase reprodutiva foram determinados os períodos de pré-oviposição, oviposição, pós-oviposição, e o número diário e total de ovos colocados pelas fêmeas.

O delineamento experimental foi o inteiramente casualizado (DIC), constituído, por oito tratamentos (dietas) e 10 repetições, sendo cada repetição representada por 10 lagartas de $H$. armigera mantidas na dieta e, sequencialmente, 1 casal de adultos de $H$. armigera representando a repetição. Os dados originais foram submetidos ao teste de homocedasticidade e à análise de variância. Quando foi constatado efeito significativo de tratamento, as médias foram comparadas pelo teste de Skott-Knott a $5 \%$ de probabilidade. Para comparação da adequação das diferentes dietas na criação de $H$. armigera, foi utilizado a tabela de vida e de fertilidade, considerando-se as repetições das fases imaturas que atingiram a fase adulta baseandose em Silveira Neto et al. (1979). Os resultados obtidos na tabela de vida foram também submetidos a análise de variância e os parâmetros comparados estatisticamente pelo teste de Skott-Knott $(p<0,05)$.

A porcentagem de contaminação nos tubos de dieta artificial de criação de lagartas de $\mathrm{H}$. armigera foi menor nos tratamentos com formaldeído $(4,5 \mathrm{~mL})$ e para as maiores concentrações de ácido sórbico $(3,7 \mathrm{~g})$ e de ácido benzóico $(3,7 \mathrm{~g})$, seguidos pelo tratamento contendo a maior dose de Ácido acetilsalićlico (2g) (Fig. 1). No entanto nesse caso, não ocorre lavagem dos ovos, ou seja, a desinfecção com sulfato de cobre como e comumente recomendado pela literatura para metodologia de criação de $D$. saccharalis, o que, possivelmente, contribuiu para a maior contaminação observada na dieta de criação de $H$. armigera nos tratamentos onde haviam menor doses/ concentrações dos produtos testados. Os anticontaminantes avaliados neste estudo têm sido comumente referidos na literatura para uso em dietas artificiais de criação de insetos (Parra \& Zucchi 1997; Alverson \& Conhen 2002; Parra 2015).

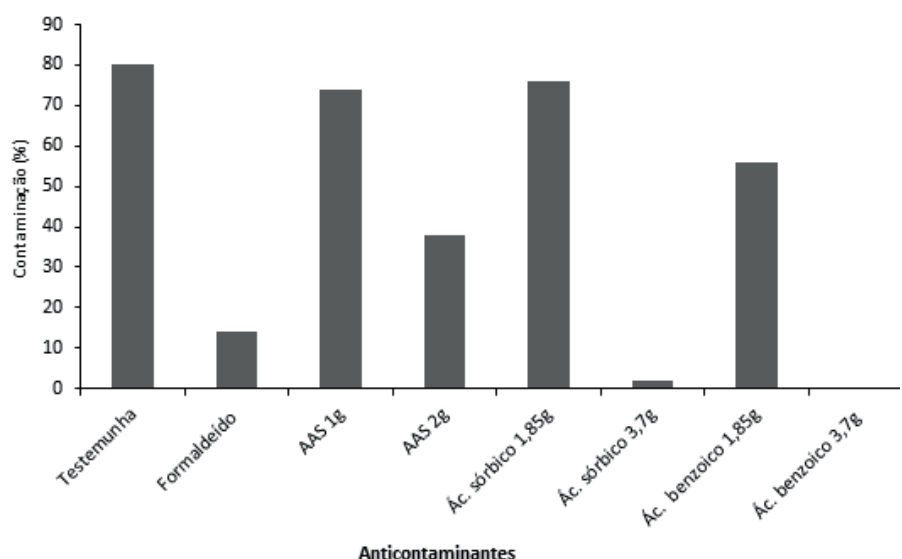

Figura 1. Contaminação de dieta artificial com diferentes anticontaminantes para criação de $H$. armigera em laboratório. AAS: Ácido acetilsalicílico

O período embrionário de $H$. armigera foi maior nos tratamentos com ácido sórbico em ambas as concentrações estudadas, enquanto nos demais tratamentos o período embrionário foi semelhante aquele observado na testemunha (Tab. 2). Segundo Dunkel \& Read (1991) algumas famílias da ordem Lepidoptera podem ser afetadas pelo ácido sórbico, constatando efeitos negativos, principalmente para a duração do para a fase de ovo, semelhante aos resultados deste estudo. A viabilidade da fase de ovo não foi influenciada pelos diferentes tratamentos aplicados na dieta artificial (Tab. 2). A viabilidade dessa fase não foi influenciada pelo uso de anticontamminantes. Em contrapartida Sikorowski \& Thompson (1984) ao estudar bactérias contaminantes em dieta artificial para criação de $C$. virescens, observaram efeitos prejudiciais dos anticontaminantes testados na eclosão dos ovos.

A duração da fase larval foi semelhante entre os diferentes tratamentos aplicados na dieta, enquanto que a viabilidade dessa fase de desenvolvimento foi significativamente maior nos tratamentos com as maiores concentrações de ácido sórbico e ácido benzóico (Tab. 2 ), enquanto nos demais tratamentos a viabilidade da fase larval foi menor, diferindo da testemunha, que foi o tratamento que mais afetou a viabilidade de lagartas, evidenciando que a contaminação 
Tabela 2. Duração (dias) e viabilidade (\%) das fases de ovo, lagarta e pupa de $H$. armigera em dieta artificial com diferentes anticontaminantes.

\begin{tabular}{|c|c|c|c|c|c|c|}
\hline \multirow{2}{*}{ Anticontaminantes } & \multicolumn{3}{|c|}{ Duração (dias) } & \multicolumn{3}{|c|}{ Viabilidade (\%) } \\
\hline & Ovo & Lagarta & Pupa & Ovo & Lagarta & Pupa \\
\hline Testemunha & $5,15 \pm 0,2 b^{1}$ & $22,09 \pm 0,9$ a & $14,61 \pm 0,8$ a & 38,8 a & $38,0 \mathrm{c}$ & $72,17 \mathrm{a}$ \\
\hline Formaldeído & $5,30 \pm 0,2 b$ & $21,09 \pm 0,4$ a & $16,61 \pm 0,8$ a & $32,0 \mathrm{a}$ & $68,0 \mathrm{~b}$ & 92,55 a \\
\hline Ácido acetilsalicílico (1g) & $4,95 \pm 0,4 b$ & $22,11 \pm 0,8$ a & $16,19 \pm 1,0$ a & $35,2 \mathrm{a}$ & $60,0 \mathrm{~b}$ & $82,07 \mathrm{a}$ \\
\hline Ácido acetilsalicílico $(2 \mathrm{~g})$ & $5,10 \pm 0,0 \mathrm{~b}$ & $21,82 \pm 0,9$ a & $15,13 \pm 0,6$ a & $38,3 \mathrm{a}$ & $61,0 \mathrm{~b}$ & $65,45 a$ \\
\hline Ácido sórbico (1,85g) & $5,75 \pm 0,2$ a & $21,95 \pm 0,8$ a & $15,31 \pm 0,4$ a & $26,9 \mathrm{a}$ & $62,0 \mathrm{~b}$ & 80,07 a \\
\hline Ácido sórbico $(3,7 \mathrm{~g})$ & $5,55 \pm 0,2 a$ & $20,23 \pm 0,2$ a & $16,33 \pm 0,2$ a & $52,1 \mathrm{a}$ & $82,0 \mathrm{a}$ & $81,41 \mathrm{a}$ \\
\hline Ácido benzóico $(1,85 \mathrm{~g})$ & $5,15 \pm 0,2 b$ & $22,46 \pm 1,5$ a & $15,29 \pm 0,8$ a & $41,9 a$ & $59,0 \mathrm{~b}$ & $84,30 \mathrm{a}$ \\
\hline Ácido benzóico $(3,7 \mathrm{~g})$ & $5,00 \pm 0,0 \mathrm{~b}$ & $20,43 \pm 0,5$ a & $16,74 \pm 0,4$ a & $44,1 \mathrm{a}$ & 74,0 a & $88,04 \mathrm{a}$ \\
\hline
\end{tabular}

${ }^{1}$ Médias \pm EP seguidas pela mesma letra na coluna não diferem estatisticamente, entre si, pelo teste de Skott-Knott $(p<0,05)$.

da dieta pode prejudicar o desenvolvimento dos insetos. Segundo Eduardo et al. (2018) a melhor sanidade de uma dieta pode ser um fator de fagoestimulação, que proporciona uma maior qualidade e ingestão de alimento, promovendo a produção de insetos com melhor qualidade. Sikorowski \& Thompson (1984) estudaram o efeito de microorgaanismos contaminantes em dietas artificial para criação de H. virescens e observaram uma diminuição significativa do ácido úrico detectada no sangue desse inseto, indicando aumento da excreção de água nas fezes. Verificou também um aumento significativo inexplicado de colesterol no sangue das larvas quando contaminadas.

A duração e a viabilidade da fase de pupas não foram afetadas pelo uso dos anticontaminantes na dieta bem como não apresentaram diferenças do tratamento testemunha (Tab. 2), evidenciando que os anticontaminantes aplicados na dieta artificial não interferem no desenvolvimento e na sobrevivência deste estágio de desenvolvimento de $H$. armigera. Os pesos de pupas machos e de fêmeas, não foram influenciados pelos diferentes tratamentos aplicados na dieta artificial (Tab. 3), bem como a longevidade de machos e de fêmeas de adultos de $H$. armigera que foram semelhantes em todos os tratamentos do ensaio (Tab. 3). Com relação as características biológicas avaliadas durantes a fase reprodutiva de $H$. armigera, tais como período de pré-oviposição, de oviposição e de pós-oviposição, bem como a fecundidade diária e total de ovos colocados por fêmea não foram influenciados pelo uso dos anticontaminantes na dieta, sendo todos semelhantes quando comparados ao tratamento testemunha (Tab. 4).

A taxa líquida de reprodução (Ro) de fêmeas de $H$. armigera foi maior para os insetos alimentados com dietas contendo formaldeído, seguido pelos tratamentos com ambas as concentrações de ácido benzóico e com a maior concentração de ácido sórbico, os quais superaram todos os demais tratamentos, em especial a testemunha que apresentou a menor taxa líquida de reprodução (Tab. 5). Já a taxa intrínseca de crescimento $(\mathrm{Rm})$ e a razão finita de aumento $(\lambda)$ foram maiores no tratamento com formaldeído e naquele com a maior concentração de ácido benzóico, enquanto o menor valor desses dois parâmetros foi verificado na testemunha, que apresentou novamente o menor valor, sendo significativamente inferior a todos os demais tratamentos do ensaio (Tab. 5). O intervalo de tempo entre as gerações (T) de $H$. armigera foi inferior com ácido benzóico em ambas as concentrações testadas, não diferindo daquele observado com a menor concentração de ácido sórbico e com a testemunha, enquanto os demais tratamentos apresentaram valores de T superiores (Tab. 5). Quanto o tempo gasto para a população de $H$. armigera duplicar em número (TD), verificouse que os menores valores foram observados nos tratamentos com formaldeído, com ácido benzóico, nas duas concentrações testadas e com a menor de ácido sórbico ao passo que o maior TD foi observado no tratamento testemunha (Tab. 5).

O tratamento testemunha, talvez devido à baixa sobrevivência observada durante a fase larval em decorrência da contaminação, foi aquele mais prejudicado nas características da tabela de vida, seguido por aqueles tratamentos com ácido acetilsalicílico. Os tratamentos contendo ácido benzóico e sórbico destacaram-se por apresentarem maior crescimento dos indivíduos dentro desses respectivos grupos. As maiores doses dos produtos testados podem substituir o formaldeído na dieta por assegurarem maior eficácia no controle de microorganismos. Todavia, dentre os diferentes anticontaminates estudados considera-se o ácido benzóico na maior concentração testada $(3,7 \mathrm{~g})$ como o mais adequado para criação de $H$. armigera.

\section{Agradecimentos}

Os autores agradecem a Universidade Federal da Grande Dourados (UFGD), Embrapa Agropecuária Oeste e Coordenação de Aperfeiçoamento de Pessoal de Nível Superior (CAPES).

Tabela 3. Peso de pupas (macho e fêmea) e longevidade de adultos (macho e fêmea) oriundos de lagartas de $H$. armigera criadas em dieta artificial com diferentes anticontaminantes.

\begin{tabular}{|c|c|c|c|c|}
\hline \multirow{2}{*}{ Anticontaminantes } & \multicolumn{2}{|c|}{ Peso de pupas (g) } & \multicolumn{2}{|c|}{ Longevidade (dias) } \\
\hline & Macho & Fêmea & Macho & Fêmea \\
\hline Testemunha & $0,397 \pm 0,03 a^{1}$ & $0,378 \pm 0,02$ a & $6,2 \pm 0,5$ a & $8,5 \pm 0,9 a$ \\
\hline Formaldeído & $0,416 \pm 0,01 \mathrm{a}$ & $0,399 \pm 0,01 \mathrm{a}$ & $9,7 \pm 2,4$ a & $9,7 \pm 0,2$ a \\
\hline Ácido acetilsalicílico (1g) & $0,403 \pm 0,01 \mathrm{a}$ & $0,367 \pm 0,03 \mathrm{a}$ & $9,0 \pm 1,3$ a & $8,8 \pm 1,3$ a \\
\hline Ácido acetilsalicílico (2g) & $0,401 \pm 0,03$ a & $0,388 \pm 0,02 \mathrm{a}$ & $9,1 \pm 0,8$ a & $7,1 \pm 0,9$ a \\
\hline Ácido sórbico $(1,85 \mathrm{~g})$ & $0,409 \pm 0,03 \mathrm{a}$ & $0,414 \pm 0,00 \mathrm{a}$ & $7,2 \pm 2,3 \mathrm{a}$ & $9,2 \pm 1,4 \mathrm{a}$ \\
\hline Ácido sórbico (3,7g) & $0,420 \pm 0,02 \mathrm{a}$ & $0,404 \pm 0,01$ a & $6,0 \pm 1,9$ a & $7,9 \pm 2,5 \mathrm{a}$ \\
\hline Ácido benzóico (1,85g) & $0,401 \pm 0,01 \mathrm{a}$ & $0,389 \pm 0,05 \mathrm{a}$ & $9,6 \pm 2,0 \mathrm{a}$ & $8,4 \pm 0,8 \mathrm{a}$ \\
\hline Ácido benzóico (3,7g) & $0,407 \pm 0,02 a$ & $0,402 \pm 0,02 a$ & $8,1 \pm 1,5$ a & $9,1 \pm 2,4 \mathrm{a}$ \\
\hline
\end{tabular}

${ }^{1}$ Médias \pm EP seguidas pela mesma letra na coluna não diferem estatisticamente, entre si, pelo teste de Skott-Knott $(p<0,05)$. 
Tabela 4. Duração (dias) dos períodos de pré-oviposição, oviposição e pós-oviposição; e fecundidade diária e total de $H$. armigera criadas em dieta artificial com diferentes anticontaminantes.

\begin{tabular}{|c|c|c|c|c|c|}
\hline \multirow{2}{*}{ Anticontaminantes } & \multirow{2}{*}{ Pré-oviposição } & \multirow{2}{*}{ Oviposição } & \multirow{2}{*}{ Pós-oviposição } & \multicolumn{2}{|c|}{ Fecundidade } \\
\hline & & & & Diária & Total \\
\hline Testemunha & $2,4 \pm 0,2 \mathrm{a}$ & $4,9 \pm 0,4$ a & $2,0 \pm 1,0 \mathrm{a}$ & $78,28 \pm 28,3$ a & $456,60 \pm 217,0 \mathrm{a}$ \\
\hline Formaldeído & $2,1 \pm 0,3$ a & $5,7 \pm 0,8$ a & $1,7 \pm 0,3$ a & $113,51 \pm 9,8 \mathrm{a}$ & $603,80 \pm 85,9$ a \\
\hline Ácido acetilsalicílico (1g) & $2,3 \pm 0,5 \mathrm{a}$ & $4,4 \pm 0,6$ a & $2,4 \pm 0,5 \mathrm{a}$ & $60,67 \pm 19,6$ a & $220,10 \pm 87,8$ a \\
\hline Ácido acetilsalicílico (2g) & $3,4 \pm 0,4 \mathrm{a}$ & $3,9 \pm 0,3$ a & $1,3 \pm 0,6 a$ & $122,10 \pm 64,2 \mathrm{a}$ & $456,70 \pm 137,0$ a \\
\hline Ácido sórbico (1,85g) & $2,6 \pm 0,3$ a & $5,8 \pm 1,4$ a & $1,1 \pm 0,3$ a & $80,15 \pm 31,9$ a & $486,80 \pm 210,9 a$ \\
\hline Ácido sórbico $(3,7 g)$ & $3,1 \pm 0,2$ a & $4,1 \pm 0,5$ a & $1,9 \pm 0,3 a$ & $78,18 \pm 29,0$ a & $349,30 \pm 131,6$ a \\
\hline Ácido benzóico $(1,85 \mathrm{~g})$ & $2,6 \pm 0,7 \mathrm{a}$ & $4,8 \pm 0,5$ a & $1,2 \pm 0,4 \mathrm{a}$ & $122,57 \pm 54,9 a$ & $540,17 \pm 22,8$ a \\
\hline Ácido benzóico $(3,7 \mathrm{~g})$ & $3,8 \pm 0,8$ a & $4,4 \pm 0,9 a$ & $2,6 \pm 0,4 a$ & $93,84 \pm 26,5$ a & $380,70 \pm 188,7 a$ \\
\hline
\end{tabular}

'Médias E EP seguidas pela mesma letra na coluna não diferem estatisticamente, entre si, pelo teste de Skott-Knott $(p<0,05)$

Tabela 5. Taxa líquida de reprodução $(R o)$, capacidade inata de aumentar em número $(R m)$, razão finita de aumento $(\lambda)$, tempo entre cada geração (T) e tempo de duplicação da população) (TD), de H. armigera quando criada em dieta artificial com diferentes anticontaminantes.

\begin{tabular}{|c|c|c|c|c|c|}
\hline Anticontaminantes & $\mathrm{R}_{\mathrm{o}}$ & $\mathrm{R}_{\mathrm{m}}$ & $\lambda$ & $\mathrm{T}$ & TD \\
\hline Testemunha & $27,31 \mathrm{e}$ & $0,087 \mathrm{e}$ & $1,090 \mathrm{~d}$ & $34,51 b$ & $7,56 \mathrm{a}$ \\
\hline Formaldeído & $183,22 \mathrm{a}$ & $0,140 \mathrm{a}$ & $1,150 \mathrm{a}$ & $36,08 \mathrm{a}$ & $4,79 \mathrm{e}$ \\
\hline Ácido acetilsalicílico (1g) & $78,05 \mathrm{~d}$ & $0,118 d$ & $1,280 \mathrm{c}$ & $35,80 \mathrm{a}$ & $5,70 \mathrm{~b}$ \\
\hline Ácido acetilsalicílico (2g) & $86,82 \mathrm{~d}$ & $0,120 d$ & $1,290 \mathrm{c}$ & $35,82 \mathrm{a}$ & $5,52 \mathrm{c}$ \\
\hline Ácido sórbico $(1,85 \mathrm{~g})$ & $126,26 \mathrm{c}$ & $0,135 b$ & $1,440 \mathrm{~b}$ & $34,30 \mathrm{~b}$ & $4,93 \mathrm{e}$ \\
\hline Ácido sórbico $(3,7 g)$ & $137,77 \mathrm{~b}$ & $0,131 c$ & $1,410 b$ & $35,90 \mathrm{a}$ & $5,05 \mathrm{~d}$ \\
\hline Ácido benzóico $(1,85 \mathrm{~g})$ & $145,62 \mathrm{~b}$ & $0,136 b$ & $1,450 \mathrm{~b}$ & $35,19 b$ & 4,89 e \\
\hline Ácido benzóico $(3,7 \mathrm{~g})$ & $145,06 \mathrm{~b}$ & 0,139 a & 1,490 a & $34,86 \mathrm{~b}$ & $4,85 \mathrm{e}$ \\
\hline
\end{tabular}

\section{Contribuições dos autores}

Conceptualização: Moreira, S. C. S.; Silva, I. F.; Ávila, C. J.; Oliveira, H. N. Desenvolvimento de bioensaios: Moreira, S.C.S.; Silva, I. F.; Redação e edição: Moreira, S. C. S.; Silva, I. F.; Ávila, C. J.; Oliveira, H. N. Análises dos dados: Moreira, S. C. S.; Silva, I. F.; Ávila, C. J.; Oliveira, H. N.; Correção do rascunho final: Moreira, S. C. S.; Silva, I. F.; Ávila, C. J.; Oliveira, H. N.

\section{Referências}

ANVISA (2017) Agência Nacional de Vigilância Sanitária. Cosmético. http://portal.anvisa.gov.br/cosmeticos, acessado em 10.i.2017.

Alverson, J.; Conhen. A. C. (2002) Effect of Antifungal Agents on Biological Fitness of Lygus Hesperus (Heteroptera: Miridae). Journal of Economic Entomology, 95(2): 256- 260.

Ávila, C. J.; Vivan L. M.; Tomquelski G. V. (2013) Ocorrência, aspectos biológicos, danos e estratégias de manejo de Helicoverpa armigera (Hübner) (Lepidoptera: Noctuidae) nos sistemas de produção agrícolas. Dourados: Embrapa Agropecuária Oeste. 12p. (Circular Técnica, 23).

Czepak, C.; Albernaz K. C.; Vivan L. M.; Guimarães H. O.; Carvalhais, T. (2013) Primeiro registro de ocorrência de Helicoverpa armigera (Hübner) (Lepidoptera: Noctuidae) no Brasil. Pesquisa Agropecuária Tropical, 43(1): 110-113.

Dunkel, F. V.; Read, N. R. (1991) Review of the Effect of Sorbic Acid on Insect Survival in Rearing Diets with Reference to Other Antimicrobials. American Entomologist, 37(3): 172-178.

Eduardo, W. L.; Moraes, R. F. O.; Almeida, L. F. V.; Santos, J. A.; Santos, R. F.; Bortoli, S. A. (2018) Alternative to formaldehyde in artificial diet for sugarcane borer and its effects on the parasitoid. Comunicata Scientiae, 8(2): 194-201. doi: 10.14295/cs.v8i1.1740

Greene, G. L.; Leppla, N. C.; Dickerson, W. A. (1976) Velvet bean caterpillar: a rearing procedure and artificial medium. Journal of Economic Entomology, 69(4): 487-488.

Hensley, S. D.; A. Hammond, H. (1968) Laboratory techniques for rearing the sugar cane borer on an artificial diet. Journal of Economic Entomology, 61(3): 1742-1743.
Kasten, J. R. P.; Precetti, A. A. C. M.; Parra, J.R.P (1978) Dados biológicos comparativos de Spodoptera frugiperda (J.E. Smith, 1797) em duas dietas artificiais e substrato natural. Revista de Agricultura, 53(3):69-78.

Moreti, A. C. C. C., Parra, J. R. P. (1983) Biologia comparada e controle de qualidade de Heliothis virescens (Fabr., 1781) (Lepidoptera: Noctuidae) em dietas natural e artificial. Arquivos do Instituto Biológico, 50(4):7-15.

Nicrom Química (2017) Ficha de Informação e Segurança do Produto Químico Formaldeído. http://nicromquimica.com.br/ produtoscosmeticos/\#wpcf7-f156-p597. Acessado em 10.i.2017.

Oswaldo Cruz. (2017) Ácido acetilsalicílico. https://www.oswaldocruz. br/download\%C3\%81cido\%20acetilsalic\%C3\%ADlico2003.pdf. Acessado em 24.i.2017.

Parra, J. R. P.; Zucchi, R. A. (1997) Trichogramma e o controle biologico aplicado. Piracicaba: ESALQ/FEALQ SP.

Parra, J. R. P. (2015) Técnicas de criação de insetos para programas de controle biológico. Piracicaba: ESALQ/FEALQ.

Razmjou, J.; Naseri, B. (2014) Comparative performance of the cotton bollworm, Helicoverpa armigera (Hübner) (Lepidoptera: Noctuidae) on various host plants. Journal Pest Science, 87: 29-37. doi: 10.1007/s10340-013-0515-9

Sikorowski, P. P.; Thompson, A. C. (1984) Effects of bacterial contamination on development and blood chemistry of Heliothis virescens. Comparative Biochemistry and Physiology Part A: Physiology, 77(2): 283-285.

Silva, I. F.; Baldin, E. L. L.; Specht, A.; Sosa-Gómez, D. R.; Roque-Specht, V. F.; Morando, R.; Paula-Moraes, S. V. (2018) Biotic potential and life table of Helicoverpa armigera (Hübner) (Lepidoptera: Noctuidae) from three brazilian regions. Neotropical Entomology, 47(3): 344-351. doi: 10.1007/s13744-017-0529-8

Silveira Neto, S.; Nakano, O.; Barbin, D.; Villa Nova, N. A. (1979) Manual de ecologia dos insetos. Piracicaba: Ceres.

Smith, C. N. (1996) Insect colonization and mass production. New York; Academic. 